WellBeing International

WBI Studies Repository

3-1995

\title{
Indicating Acts During Counting by a Chimpanzee (Pan troglodytes)
}

Sarah T. Boysen

Ohio State University

Gary G. Berntson

Ohio State University

Traci A. Shreyer

Ohio State University

Michelle B. Hannan

The Ohio State University

Follow this and additional works at: https://www.wellbeingintlstudiesrepository.org/acwp_asie

Part of the Animal Studies Commons, Comparative Psychology Commons, and the Other Animal Sciences Commons

\section{Recommended Citation}

Boysen, S. T., Berntson, G. G., Shreyer, T. A., \& Hannan, M. B. (1995). Indicating acts during counting by a chimpanzee (Pan troglodytes). Journal of Comparative Psychology, 109(1), 47.

This material is brought to you for free and open access by WellBeing International. It has been accepted for inclusion by an authorized administrator of the WBI Studies Repository. For more information, please contact wbisr-info@wellbeingintl.org.

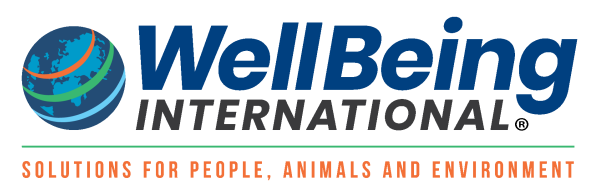




\title{
Indicating Acts During Counting by a Chimpanzee (Pan troglodytes)
}

\author{
Sarah T. Boysen ${ }^{1,2}$, Gary G. Berntson ${ }^{1,2}$, Traci A. Shreyer ${ }^{1}$, and Michelle B. Hannan ${ }^{1}$ \\ ${ }^{1}$ Ohio State University \\ ${ }^{2}$ Yerkes Regional Primate Research Center
}

\begin{abstract}
$\underline{\text { ABSTRACT }}$
A chimpanzee (Pan troglodytes) experienced in counting arrays of 0-7 items and trained for comprehension of number symbols, spontaneously displayed a variety of indicating acts (e.g., pointing, touching, and rearranging items) during counting. Twenty-five sessions were videotaped, and all trials were evaluated for the relations among number of items presented, number of indicating acts displayed, and the Arabic number selected to represent the array. Significant correlations included the relations between number of items and the cardinal number selected by the animal, between the number of items and indicating acts displayed by the chimpanzee, and between the number of indicating acts and the numeral selected. These data suggest that the use of indicating acts by this animal may have functional significance and serves as an organizing schema, comparable to similar behaviors observed in children in the early stages of learning to count.
\end{abstract}

In recent years, innovative approaches to the study of animal behavior and cognition in captivity have provided evidence for complex abilities that often do not appear to be adaptively necessary for the functional, biological success of a particular species in its natural environment (e.g., Boysen \& Berntson, 1989; Davis, 1984; Gisiner \& Schusterman, 1992; Herman, Morrel-Samuels, \& Pack, 1990; Matsuzawa, 1985; Pepperberg, 1987). Nevertheless, these and other studies have provided new insights into possible relations between learning capacity, a species' natural history, and the phylogeny of cognition. For instance, given the significant impact of time, space, and number on the day-to-day survival of many species (Gallistel, 1989), the capacity for number concepts and counting may not reflect a totally artificial capability in some nonhuman species. In this regard, an important question arises as to whether the numerical capabilities of animals evidence an evolutionary continuity with counting in humans or, rather, reflect fundamentally different processes and mechanisms. Some insight into this issue may be gained by behavioral features or correlates of counting that emerge de novo, without explicit training.

Young children learning to count display a range of behavioral acts to mediate between words spoken in time and an array of objects that are distributed in space (Fuson, 1988; Gelman \& Gallistel, 1978). They typically use several behaviors for such mediation, termed indicating acts (Fuson, 1988), to organize array items. These processes have also been termed partitioning and tagging (Gelman \& Gallistel, 1978) and entail the physical acts associated with the indicating feature (partitioning) and the labelling (verbal or otherwise) of individual items (tagging; see Davis \& Perusse, 1988, and Thomas \& Lorden, 1993, for discussion). For correct counting to occur, items must be counted or tagged only once. However, as 
children's expertise with counting is emerging, numerous error types that result in inaccurate counts occur. Such errors may result, for example, in the incorrect assignment of a cardinal number as the label for the array. These violations appear to be features of the normal development of counting skills in young children, and thus the use of indicating acts may serve to help them separate items in an array that have already been counted and those that remain to be counted (Fuson, 1988; Gelman \& Gallistel, 1978).

Tagging or indicating behaviors have also been observed and reported anecdotally in a limited number of early studies of counting in animals (see Davis \& Perusse, 1988, for review). That indicating acts may serve a mediation function was first noted by Koehler (1950) for birds and Mechner (1958) with rats. Koehler described how a jackdaw returned to a trial that had not been completed (the bird was to select only 5 baits from among several available in boxes) and ceremoniously bowed to each box exactly the number of times equal to the number of food items it had just removed from each box. Similarly, Mechner described the behavior of a rat trained on a fixed consecutive number schedule during which the rat was reinforced for presses on one lever and then required to indicate completion of responses to the first lever by pressing a second lever. Mechner noted that one rat consistently used its right paw to press the first lever while simultaneously completing a semicircle on the wall with its left paw, after which it depressed the second lever.

Davis and Memmott (1982) provided convincing evidence that these topographic chains did not likely function in a manner that was analogous to the use of a verbal number sequence in human counting. Davis and Perusse (1988) further suggested that although earlier instances of overt analogues to verbal labels, whether they were behavioral or motor tags, appeared to be "tantalizing evidence" (p. 566), verbal number tags did not have to be replaced by physical markers in nonhuman species. They argued that this position was consistent with the conclusions put forth by Gelman and Gallistel (1978), who described tags as potentially consisting of either overt or covert behaviors.

For several years, we have been exploring the capacity of the chimpanzee (Pan troglodytes) to acquire facility with counting and other number-related skills (Boysen, 1993; Boysen \& Beratson, 1989). In view of the importance of indicating acts in children during the acquisition of counting skills, and the possibility of continuity in counting processes of children and other nonhuman species, the identification of such behaviors during the acquisition of counting skills in chimpanzees may be of considerable relevance to the origins and phylogeny of numerical abilities. We report the spontaneous display of indicating acts by a young chimpanzee during counting training sessions in which food items served as stimuli. Preliminary observations suggested that these behaviors might be serving an important function for this animal and that such indicating acts might be related to those observed in very young children as they first learned to count (Fuson, 1988; Gelman \& Gallistel, 1978; Saxe \& Kaplan, 1981). The purpose of this study was to evaluate the relation between the number of items presented for counting, the number of item-directed behaviors exhibited by the animal, and the Arabic numeral the chimpanzee selected to represent the array.

\section{Method}

\section{Subject}

The subject was a 9-year-old female chimpanzee (Pan troglodytes), Sheba, who was human crossfostered for the first $2 \frac{1}{2}$ years of life and was on permanent loan from the Columbus Zoo. Sheba had been in direct social and physical contact with her human teachers and other chimpanzees daily since her arrival. She had also been immersed in training on numerous cognitive tasks (e.g., Boysen \& Berntson, 1989; Boysen, Berntson, Shreyer, \& Quigley, 1993). Sheba was housed in a social group with two other chimpanzees, including a 28-year-old female and 3-year-old male. All six chimpanzees in the colony lived 
in an enriched environment, with continual year-round access to indoor and outdoor play areas equipped with toys and climbing structures.

\section{Procedures}

Daily counting training consisted of 20 - to 30 -min sessions, with $18-25$ trials per session. Sheba was unrestrained during all sessions and was free to move about the testing area. Typically, Sheba was highly compliant and attentive during testing and had been working with the same teacher (Sarah T. Boysen) since 1984. No food deprivation was used; Sheba was maintained on a daily diet that consisted of a wide variety of fresh fruits, vegetables, and treats over 3-5 feedings. All training and testing sessions were completed between $1,000 \mathrm{hr}$ and $1,500 \mathrm{hr}$. During the counting task small candies (chocolates or gumdrops) were used as stimuli and then given as reinforcement once they were counted by Sheba.

During testing, Sheba and the experimenter were either seated on the floor or at a large table, and all counting sessions were videotaped for later analysis. The experimenter provided Sheba with a series of $7.5 \times 12.5 \mathrm{~cm}$ plastic placards bearing the Arabic numerals 0-7. During previous training and testing, numerals were explicitly randomized to avoid position bias, as reported in Boysen and Berntson (1989). Hence, the chimpanzees had no history of association between symbols and spatial loci but rather had considerable training that explicitly dissociated symbol meaning from spatial location. In this study, because our principal interest was in the physical indicating behaviors exhibited by Sheba, number symbols were maintained in ordinal sequence to maximize Sheba's ability to quickly locate the number, with minimal attentional demands.

It is important to note that indicating behaviors were never explicitly reinforced. However, when Sheba was first learning number concepts, she made frequent errors in assigning the correct cardinal number to represent an array. When she was wrong, she was given a second opportunity to count the objects and choose the correct number symbol. If she failed on the correction trial, the experimenter pointed to and usually touched each item as she counted, while she simultaneously said each count word in synchrony with the indicating behaviors. After all items were noted, the experimenter pointed to the correct Arabic numeral placard and repeated the correct number label verbally.

In this study, as the items were presented, the experimenter asked verbally, "How many is this?" Sheba was required to touch, or point to, the number placard with the correct Arabic numeral that corresponded to the size of the candy array. A video camera was positioned directly over the numerals and both permitted monitoring of the task so that the chimpanzee was unable to see the experimenter and provided a permanent video record of each trial. If Sheba chose an incorrect number, she was given the opportunity to try again and in most cases was correct on the second trial. However, only Sheba's first choice on a given trial was scored. When correct, she was given the candies from the array. The experimenter's judgment of responses was used only for immediate reward feedback, with formal data analyses based on off-line analysis of videotapes.

We have demonstrated in previous studies that Sheba could correctly select the Arabic symbol that matched the number of items in an array (Boysen \& Berntson, 1989). In this study, we were primarily interested in Sheba's indicating behaviors, their relation to the number of items presented on each trial, and the Arabic numeral Sheba chose to represent each array. However, we also scored her response as the first number selected on each trial, as evaluated by two independent judges.

\section{Videotape Analysis of Counting Sessions}

All videotaped counting sessions were scored by two observers who were unaware of the experimental hypotheses. During the first pass through the tapes, a behavioral glossary that included the range of 
indicating acts exhibited was devised. Next, observers reviewed the tapes again and recorded the number of indicating acts that Sheba directed toward the array items. Sheba frequently maintained contact with the items while touching, pushing, or moving the item around but sometimes momentarily lost contact with the candy. In other instances, the indicating act consisted of two or more staccatolike taps to the item. In both cases, however, the indicating event entailed a coherent, uninterrupted stream of behavior that differed from other cases in which discrete indicating behaviors were displayed to the same (or different) items. A single indicating act was scored if Sheba contacted an item, removed her hand, and immediately replaced it on the same item within $1 \mathrm{~s}$ or if Sheba pushed an item to another area of the tray and then touched it. All other repetitive contacts were scored as separate indicating acts. The independent observers were able to reliably distinguish these response classes, as evidenced by the high degree of interrater reliability $(r=.98)$. The few instances of disagreement were evaluated and resolved by a third observer.

Table 1. Error Distribution by Array Type (0-7)

\begin{tabular}{|cccccc|}
\hline & \multicolumn{2}{c}{ No. trials } & & \multicolumn{2}{c|}{ Mean no. tags } \\
\cline { 2 - 3 } No. items in array & Correct & Total & \% age correct & Correct trials & Incorrect trials \\
\hline 0 & 34 & 36 & 94 & 0 & 0 \\
1 & 40 & 68 & 59 & 1.4 & 2.1 \\
2 & 36 & 67 & 54 & 3.6 & 5.2 \\
3 & 39 & 74 & 53 & 6.0 & 8.8 \\
4 & 25 & 66 & 38 & 7.1 & 11.1 \\
5 & 32 & 62 & 52 & 9.7 & 14.9 \\
6 & 11 & 25 & 44 & 10.6 & 18.6 \\
7 & 12 & 24 & 50 & 14.5 & 20.1 \\
Total & 229 & 422 & 54 & & \\
\hline
\end{tabular}

During the third review of the tapes, the observers recorded the number of items (candies) in the arrays for each trial, and the final pass was to record the cardinal number that Sheba chose. A small percentage of the total trials (13 of 435 , or $3 \%$ ) were excluded from analysis because Sheba failed to respond, and the trial was terminated.

\section{Results}

\section{Overall Performance}

Sheba correctly selected the Arabic numeral corresponding to the number of items in the counting array on 229 of 422 trials (54\% correct). Given eight response options (numerals $0-7$ ), chance probability was calculated to be 0.125 . A chi-square test revealed Sheba's overall performance to be highly significant, in relation to chance, $X^{2}(1, N=422)=673.02, p<.001$. To take into consideration the possibility that some position cues from linear presentation of the numerals may have increased chance probability (e.g., by restricting attention to a subset of the response options), Sheba's performance was also evaluated with a more conservative probability (0.25). The resulting chi-square test was also significant, $X^{2}(1, N=422)=$ 192.76, $p<.001$. Errors were approximately equivalent across the 1-7 array sizes (see Table 1 ), but few errors (6\%) were made on the zero array-size condition. 
Indicating Acts

Analysis of the videotaped sessions revealed a variety of indicating acts that constituted Sheba's repertoire. These included pointing to an item without touching it, physically touching a candy without moving it, and physically displacing items in the array from their original positions and placing them a short distance away on the test tray.

Correlation and regression analyses were used to examine the relations among the number of items in the sample array, the number of indicating acts exhibited, and the Arabic numeral selected. The intercorrelations among these variables is illustrated in Table 2. Consistent with the significant performance documented earlier, the Arabic number chosen was correlated with the number of items in the stimulus array $(r=.74)$. Of greater interest is the fact that the number of indicating acts displayed by Sheba was also significantly correlated with the number of items in the stimulus array $(r=.67)$. Moreover, a significant correlation emerged between the number of indicating acts and the Arabic numeral selected as the correct count (cardinal number) for each array $(r=.52)$.

Separate correlational analyses on correct and incorrect trials (Table 2) revealed a further pattern in the interrelationships among the variables. As illustrated in Table 2, indicating acts were equally correlated with the number of items in the stimulus array for both correct and incorrect trials; that is, the number of acts tended to reflect the array size, regardless of whether the animal's response (choice of an Arabic numeral) was correct or incorrect. The primary difference between correct trials and incorrect trials was the extent to which Sheba's number selection correlated with the number of indicating acts she performed. On correct trials, as shown in Table 2, the response selection was reasonably correlated with the number of behaviors emitted $(r=.68)$. In contrast, the correlation between the number of behaviors and the animal's number selection $(r=.31, p<.05)$ was significantly lower for incorrect trials.

Table 2. Intercorrelations Between Array Size, Indicating Acts (Tags), and Numerical Selection (Response)

\begin{tabular}{|lcccc|}
\hline Trials & $\boldsymbol{n}$ & Array size-response & Array size-tags & Tags-response \\
\hline All & 422 & .74 & .67 & .52 \\
Correct & 229 & 1.00 & .68 & .68 \\
Incorrect & 193 & .32 & .67 & .31 \\
\hline
\end{tabular}

* all $p s<.01$.

Even on incorrect trials, however, Sheba's responses were systematic, and her errors were nonrandom. This is indicated by the modest but significant correlation between the number of items in the stimulus array and the number selected on incorrect trials $(r=.32)$. In fact, most response errors were off by only a single unit; that is, Sheba generally overestimated the actual number of items. Sheba also tended to overtag, in relation to the number of items in the stimulus array (see Table 2). Regression analysis revealed an approximate double indicating of items on correct trials (number of indicating acts $=-0.30+$ $1.98 \times$ Items, $R^{2}=.47, p<.001$ ). This overindicating was even more extreme on incorrect trials, for which indicating behaviors were approximately three times greater than the number of items in the stimulus array (number of indicating acts $=-1.02+3.15 \times$ Items, $R^{2}=.44, p<.001$ ). Using Fuson's (1988) taxonomy, these may represent "recount errors" (p. 67), in which a single item is counted more than once on a given trial. In contrast, Sheba appeared to make few "uncounted errors" (Fuson, 1988, p. 66), in which an item was skipped or ignored during a counting run. Because indicating acts can include pointing in the absence of direct contact, it is possible that when two items were close together, items may have been skipped. For the most part, however, Sheba's indicating behaviors suggested that this class of errors did not appear to be a prominent feature. 


\section{Discussion}

The results of this study suggest that a chimpanzee may be capable of accurately counting arrays composed of 0-7 items and that mediating motor behaviors (indicating acts), such as pointing to or moving items, may be closely associated with counting performance. Although a causal link cannot be established from correlational evidence alone, a functional contribution of these indicating acts is suggested by (a) the association between the number of acts and the number of items in the stimulus array, (b) the association between the number of acts and the chimpanzee's response selection, and (c) the significantly lower association between indicating acts and response selection on incorrect trials. The number of indicating acts on a given trial did not exactly equal the number of items in the array or the subject's response. This, however, does not rule out the possibility of functional relations among these elements, as the categorization of discrete acts based on behavioral observation may not correspond to the functional act for the animal.

It is interesting to note that the types of behaviors exhibited by Sheba during counting and her tendency to overtag at a near double rate $(1.98 \times$ Array Items) suggest the possibility that she uses indicating behaviors for both physical mediation of the counting sequence and for covert tagging, given that she is unable to verbally label each item simultaneously. She may have devised such an approach to partition and tag each item, which would require a sequential, rather than simultaneous, coordination of the two events, but which could account for her tendency to overtag. Thus, double tags may represent a type of organizing unit (indicating act and tag) that may have helped Sheba orchestrate a more accurate count. This may represent a functional parallel to the behavior of children who point to and simultaneously apply a verbal label to individual items in an array (Fuson, 1988; Gelman \& Gallistel, 1978).

There can be multiple loci of errors in this task for Sheba, including an incorrect count, mismatching of the count with the proper symbol, inaccurate motoric selection of that symbol, or attentional and memorial lapses within or between these loci. If indicating behaviors are functionally related to the animal's response selection, however, one may expect the response to be correlated with the behaviors, even if the response is incorrect. For example, if the correct answer and number of indicating acts were 4, then an erroneous response ought to be more likely to be the selection of 3 or 5 , rather than, say, 1 or 7 . That Sheba's incorrect answers, like those observed in young children (Gelman \& Gallistel, 1978), were typically off by plus or minus one lends possible support for this hypothesis.

Correspondence errors in children are subject to the effects of many variables, including age, degree of effort expended, the number of objects in the array, proximity of items to one another, the spatial organization of the array, composition of the array (homogeneous vs. heterogeneous), and the location of objects in the row (Fuson, 1988). All these factors affect both the type and number of correspondence errors that may be observed on a given trial. In relation to the age variable, most of Fuson's (1988) reported studies tested children between the ages of 3 and 6 . Not surprisingly, most errors were made by 3- to $3 \frac{1}{2} 2$-year-olds, with the rate of errors dropping considerably at age $3 \frac{1}{2}$ and continuing to decline thereafter. By age 5, children had very low error rates, with the exception of overall point-object correspondence errors during counting of disorganized arrays. In general, most errors decreased monotonically with age, which suggests that learning to count entails gradual improvement, rather than sudden and precipitous changes in skill levels. This culminates in an obvious feature of counting that occurs around age 5-6, the internalization of the counting process (Fuson, 1988).

Not surprisingly, the number of objects in the arrays presented to children also affected error rates, with larger arrays subject to increased correspondence difficulties. Younger children, in particular at ages 3 to 41/2 made more object-skipped, multiple-count, and skim errors when a larger number of items (15-29) were presented for counting. Such increases in errors were seldom seen in $4 \frac{1}{2} 2$ - to 6 -yearolds. While most 
of Fuson's (1988) subjects counted objects that were presented in immovable rows, proximity of the items was varied under some conditions. The proximity manipulation had an impact on-correspondence error rate, such that objects that were located close to one another elicited more object-skipped and multiple count errors. In addition, if objects were in close proximity to one another, children made more errors on objects that were located in the middle of the row, rather then toward the end of the row. If, however, the objects were spaced further apart, more errors were made on the last object (Fuson, 1988). In relation to the location of objects in the row, the first object was the least likely to elicit an error, and for long rows, more errors were also likely on middle objects.

The overall organization of the array was also a significant factor in affecting correspondence errors made by children. Clearly, the linear presentation of objects in the majority of these studies facilitated the correspondence process. However, when disorganized arrays (as in our study) were presented, children had greater difficulty and exhibited more correspondence errors of three types: One of every six errors were recount errors; more objects were skipped, which rarely occurred when objects were presented in rows; and the number of part word-one point errors increased. Without question, presentation of objects in a disorganized fashion made it much more difficult for children to keep track of where they began a count and which items had not yet been counted. In addition, the composition of the array also affected the type and rate of errors exhibited. If arrays were heterogeneous, more difficulties with multiple count or multiple words-one point errors were observed (Fuson, 1988). In this regard, the use of disordered and heterogeneous (different types of candies) arrays in this study may have contributed to error rates and overtagging, but these possibilities will require explicit testing.

It has been suggested that the types of indicating behaviors displayed by Sheba do not need to be demonstrated by animals in support of effective numeron use, that is, "any distinct and arbitrary tags that a mind (human or nonhuman) uses in enumerating a set of objects" (Gelman \& Gallistel, 1978, p. 77; also see Davis \& Perusse, 1988). Nonetheless, the occurrence of indicating acts by a chimpanzee may be significant as a systematic demonstration of covert behaviors by a nonhuman species during counting, which have not been previously reported. Our findings, in conjunction with other number-related skills reported for the same animal (Boysen, 1993; Boysen \& Berntson, 1989), suggest that these spontaneously occurring behaviors may have facilitated the enumeration process. The spontaneous appearance of indicating acts in a chimpanzee reveals a possible intriguing parallel between counting in this species and that of human children.

\section{References}

Boysen, S. T. (1993). Counting in chimpanzees: Nonhuman principles and emergent properties of number. In S. T. Boysen \& E. J. Capaldi (Eds.), The development of numerical competence: Animal and human models (pp. 39-59). Hillsdale, NJ: Erlbaum.

Boysen, S. T., \& Berntson, G. G. (1989). Numerical competence in a chimpanzee (Pan troglodytes). Journal of Comparative Psychology, 103, 23-31.

Boysen, S. T., Berntson, G. G., Shreyer, T. A., \& Quigley, K. S. (1993). Processing of ordinality and transitivity by chimpanzees (Pan troglodytes). Journal of Comparative Psychology, 107, 208-216.

Davis, H. (1984). Discrimination of the number "three" by a raccoon (Procyon lotor). Animal Learning and Behavior, 12, 409-413.

Davis, H., \& Memmott, J. (1982). Counting behavior in animals: A critical evaluation. Psychological Bulletin, 92, 547-571.

Davis, H., \& Perusse, R. (1988). Numerical competence in animals: Definitional issues, current evidence, and a new research agenda. Behavioral and Brain Sciences, 11, 561-615.

Fuson, K. C. (1988). Children's counting and concepts of number. New York: Springer-Verlag. 
Gallistel, C. R. (1989). Animal cognition: The representation of space, time, and number. Annual Review of Psychology, 40, 155-189.

Gelman, R., \& Gallistel, C. R. (1978). The child's understanding of number. Cambridge, MA: Harvard University Press.

Gisiner, R., \& Schusterman, R. J. (1992). Sequence, syntax, and semantics: Responses of a languagetrained sea lion (Zalophus californianus) to novel sign combinations. Journal of Comparative Psychology, 106, 78-91.

Herman, L. M., Morrel-Samuels, P., \& Pack, A. A. (1990). Bottle-nosed dolphin and human recognition of veridical and degraded video displays of an artificial gestural language. Journal of Experimental Psychology: General, 119, 215-230.

Koehler, O. (1950). The ability of birds to "count." Bulletin of Animal Behaviour, 9, 41-45.

Matsuzawa, T. (1985). Use of numbers by a chimpanzee. Nature, 315, 57-59.

Mechner, F. M. (1958). Probability relations within response sequences under ratio-reinforcement. Journal of the Experimental Analysis of Behavior, 1, 109-121.

Pepperberg, I. M. (1987). Evidence for conceptual quantitative abilities in the African Grey Parrot. Ethology, 75, 37-61.

Saxe, G. B., \& Kaplan, R. (1981). Gesture in early counting: A developmental analysis. Perceptual and Motor Skills, 53, 851-854.

Thomas, R. K., \& Lorden, R. (1993). Numerical competence in animals: A conservative view. In S. T. Boysen \& E. J. Capaldi (Eds.), The development of numerical competence: Animal and human models (pp. 127-147). Hillsdale, NJ: Erlbaum. 